\title{
Foscarnet-Resistant Cytomegalovirus Esophagitis with Stricturing
}

\author{
Vinaya Gaduputi Harish Patel Vamshidhar Vootla \\ Usman Khan Sridhar Chilimuri \\ Department of Medicine, Bronx Lebanon Hospital Center, Bronx, N.Y., USA
}

\section{Key Words}

Cytomegalovirus esophagitis · Foscarnet-resistant cytomegalovirus · Cytomegalovirus infection in HIV · Esophagitis in HIV

\begin{abstract}
We report the case of a 52-year-old man with HIV-AIDS, non-complaint with highly active antiretroviral therapy, who presented with long-standing dysphagia. He was treated for three episodes of severe Candida esophagitis with fluconazole and later caspofungin due to poor response. In spite of the prolonged treatment courses the patient did not report an improvement in his symptoms. He was also concomitantly being treated for other opportunistic infections including cytomegalovirus (CMV) retinitis with i.v. foscarnet for almost 2 months prior to the index presentation. Upper esophagogastroduodenoscopy revealed multiple superficial ulcers with stricturing. Bougie dilatation was attempted but failed. The biopsy specimens revealed multiple intracellular inclusion bodies pathognomonic of $\mathrm{CMV}$ infection. We aim to highlight the increasing resistance of $\mathrm{CMV}$ to conventional first-line antiviral agents such as foscarnet.
\end{abstract}

\section{Introduction}

Cytomegalovirus (CMV) infection of the gastrointestinal tract is one of the more uncommon opportunistic infections in AIDS [1]. The incidence of CMV infection and complications in people already afflicted with CMV has dropped precipitously after the advent of highly active antiretroviral therapy (HAART) [2-4]. Patients who are either not on HAART or are non-compliant with it present with varied and often serious manifestations of CMV infection. These manifestations include esophagitis which usually presents as multiple ulcers in the lower part [5], though an uncommon diffuse variant has been described; gastritis which usually presents with epigastric discomfort and rarely massive hemorrhage [6]; enteritis with pain and diarrhea and colitis with 
pain, diarrhea and rarely lower gastrointestinal bleeding [7]. Once the diagnosis of CMV infection of the gastrointestinal tract has been made, established treatment protocols including ganciclovir or foscarnet can be started. There have been concerning trends, however, with antiviral resistance pattern of CMV to these antiviral agents [8], either with conventional regimens or salvage regimens, which would force the clinician to adopt more toxic alternatives such as concurrent ganciclovir-foscarnet regimen [9], Foscavir or Cidofovir. Apart from their toxicity, the use of these regimens is often curtailed by factors such as poor oral bioavailability, drug resistance on prolonged use and limited efficacy [10]. Foscarnet does not require intracellular phosphorylation for antiviral activity like ganciclovir. Therefore, a UL97 phosphotransferase mutation in CMV does not usually confer resistance to foscarnet as it does to ganciclovir [11,12]. Foscarnet resistance in CMV develops after prolonged treatment for CMV retinitis often via mutations in the viral DNA polymerase gene [13]. This new drug-resistant strain of CMV can produce florid manifestations elsewhere as demonstrated in the patient we report below.

\section{Case Report}

A 52-year-old Hispanic man with HIV-AIDS, non-complaint with HAART, had multiple AIDSrelated opportunistic infections such as three prior episodes of esophageal candidiasis (confirmed with prior esophagogastroduodenoscopy, EGD) and CMV retinitis on prolonged therapy with foscarnet ( 2 months), hepatitis $\mathrm{C}$ and latent syphilis. He presented with progressively worsening dysphagia and failure to thrive with electrolyte abnormalities including severe hypokalemia $(1.7 \mathrm{mg} / \mathrm{dl})$ and hypophosphatemia $(2.2 \mathrm{mg} / \mathrm{dl})$. He was presumptively started on caspofungin for candidal esophagitis. Foscarnet therapy for CMV retinitis was stopped due to his impaired renal function but was later restarted during his hospital stay. The patient underwent EGD on day 7 of hospitalization that revealed diffuse friable mucosa in the upper third of the esophagus and a stricture $25 \mathrm{~cm}$ from the upper end of the esophagus but no evidence of candidiasis (fig. 1). Caspofungin was discontinued. EGD was repeated on day 9 of hospitalization. Neither of these proved to be complete studies or successful at dilating the stricture. Further plans of repeat EGD with bougie dilatation were aborted due to high risk of rupture of the esophagus. An esophagogram was performed instead. The esophagogram revealed a large ulceration at the junction of the upper and middle third of the esophagus with associated fusiform stricture (fig. 2). Biopsy samples taken from the area of stricture revealed intranuclear inclusions consistent with CMV esophagitis. Meanwhile the patient refused to undergo gastrostomy feeding tube placement while tolerating chopped diet better and was discharged back to his nursing home.

\section{Discussion}

This patient was severely immunocompromised and presented with dysphagia secondary to a benign-looking stricture diagnosed with EGD. The differential diagnoses of the EGD findings in this patient included HIV-related benign esophageal strictures secondary to idiopathic esophageal ulceration, herpetic esophagitis and CMV esophagitis. Benign esophageal ulcerations are unlikely in a setting of non-compliance with HAART and florid opportunistic infections elsewhere such as CMV retinitis. Herpetic ulcers usually present as well-circumscribed 'volcano-like' lesions which distinguish them from usual longitudinal and deeper ulcers seen in CMV infection. The EGD findings were more suggestive of CMV being the etiological agent especially in the setting of concomitant CMV retinitis. The diagnosis of CMV esophagitis was confirmed with biopsy. However the patient had already been on of foscarnet therapy for 
2 months prior to this presentation, highlighting the possible paradoxical presentation of a resistant strain of CMV in patients on chronic foscarnet therapy. Despite the extensive and deep nature of CMV ulceration in such cases of esophagitis, stricture formation is uncommon. It has often been noted that there is resolution of CMV esophagitis with initiation of HAART and anti-CMV agents such as ganciclovir and foscarnet. However, they can lead to fibrous healing and stricture formation [14]. Such strictures resulting from fibrous healing usually respond well to repeated esophageal dilatations, unlike in this patient in whom bougie dilatation of the CMV stricture was unsuccessful. This case is unusual at several levels, including the facts that the patient had CMV esophagitis with stricturing while on foscarnet and that the stricture was not amenable to bougie dilatation.

An increase in the resistance of CMV to conventional first-line anti-CMV agents such as ganciclovir or foscarnet has been reported, but that should not dissuade clinicians from using them for treating primary CMV infection. The secondary resistant CMV infection can be treated with one of the following strategies: sequential therapy with ganciclovir followed by foscarnet (upon failure of ganciclovir) or sequential therapy with foscarnet followed by ganciclovir or concurrent combination therapy with both foscarnet and ganciclovir $[9,15]$, with limited efficacy. Another but more toxic option is Cidofovir. It is therefore imperative that physicians be aware of more malignant presentation of CMV while patients are still on chronic conventional anti-CMV therapy and that they consider it among differential diagnoses.

\section{Disclosure Statement}

The authors declare no conflicts of interest. 


\begin{tabular}{r|l|l|l}
$\begin{array}{r}\text { Case Reports in } \\
\text { Gastroenterology }\end{array}$ & $\begin{array}{l}\text { Case Rep Gastroenterol 2013;7:25-29 } \\
\text { DOI: 10.1159/000342352 }\end{array}$ & $\begin{array}{l}\text { Published online: } \\
\text { January 10, 2013 }\end{array}$ & $\begin{array}{l}\text { @ 2013 S. Karger AG, Basel } \\
\text { ISSN 1662-0631 } \\
\text { www.karger.com/crg }\end{array}$ \\
\hline
\end{tabular}

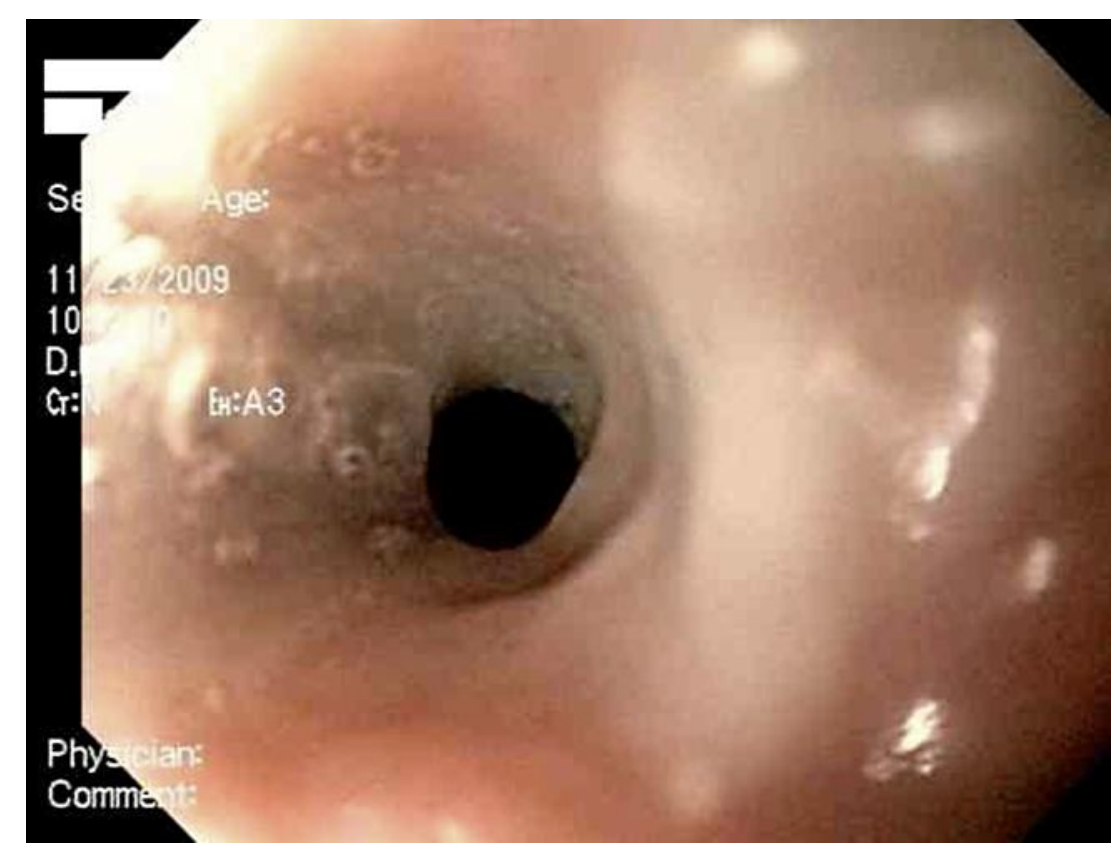

Fig. 1. Endoscopic investigation of the proximal esophagus showed multiple ulcerations and a stricture $25 \mathrm{~cm}$ from the upper end of the esophagus.

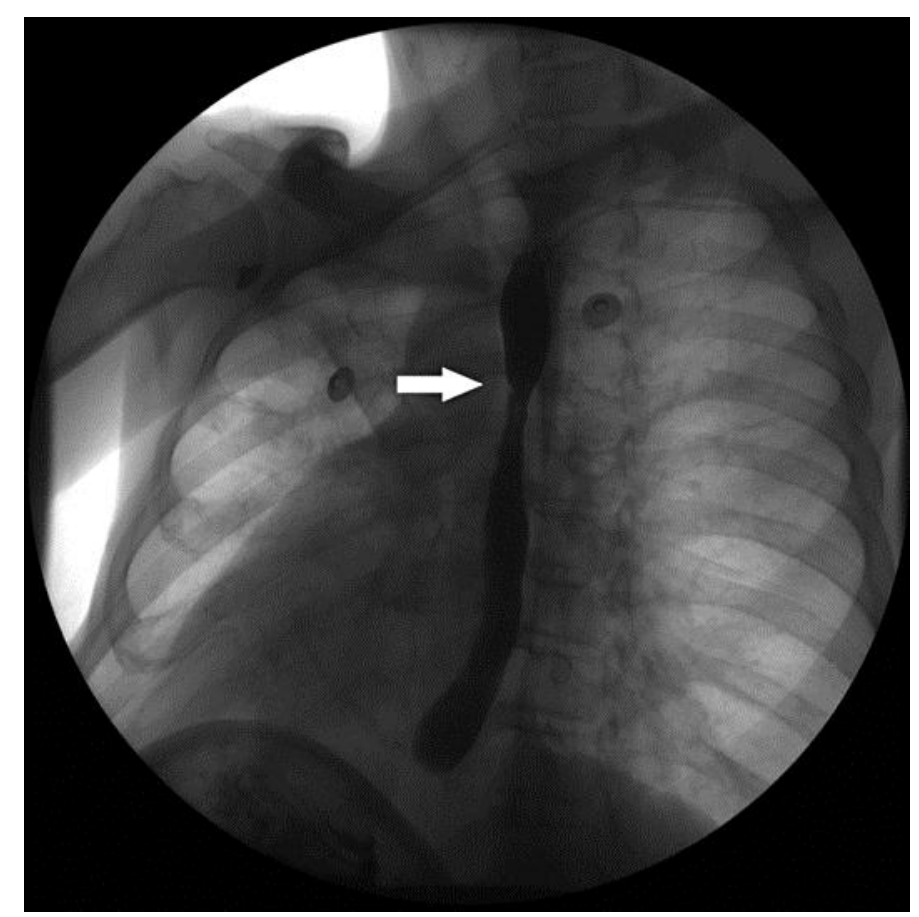

Fig. 2. Esophagogram revealed a fusiform stricture (arrow) in the upper half of the esophagus. 


\section{References}

-1 Tanaka T, Kita H: Site of action of mating factor in a-mating type cell of Saccharomyces cerevisiae. Biochem Biophys Res Commun 1978;83:1319-1324.

2 Salzberger B, Hartmann P, et al: Incidence and prognosis of CMV disease in HIV-infected patients before and after introduction of combination antiretroviral therapy. Infection 2005;33:345-349.

-3 Podlasin RB: CMV infection in HIV-infected patients (in Polish). Przegl Epidemiol 2007;61:629-637.

4 Sungkanuparph S, Chakriyanuyok T, et al: Antiretroviral therapy in AIDS patients with CMV disease: impact on the survival and long-term treatment outcome. J Infect 2008;56:40-43.

5 Wilcox CM, Straub RF, et al: Prospective endoscopic characterization of cytomegalovirus esophagitis in AIDS. Gastrointest Endosc 1994;40:481-484.

66 Vanegas F, Montalvo RD, et al: Massive upper gastrointestinal hemorrhage due to cytomegalovirus infection in two patients with acquired immunodeficiency syndrome. South Med J 2000;93:235-238.

-7 Chamberlain RS, Atkins S, et al: Ileal perforation caused by cytomegalovirus infection in a critically ill adult. J Clin Gastroenterol 2000;30:432-435.

8 Ahmed A: Antiviral treatment of cytomegalovirus infection. Infect Disord Drug Targets 2011;11: 475-503.

-9 Dieterich DT, Poles MA, et al: Concurrent use of ganciclovir and foscarnet to treat cytomegalovirus infection in AIDS patients. J Infect Dis 1993;167:1184-1188.

10 Andrei G, De Clercq E, et al: Novel inhibitors of human CMV. Curr Opin Investig Drugs 2008;9:132-145.

11 Myhre HA, Haug Dorenberg D, et al: Incidence and outcomes of ganciclovir-resistant cytomegalovirus infections in 1,244 kidney transplant recipients. Transplantation 2011;92:217-223.

12 Chou S: Cytomegalovirus UL97 mutations in the era of ganciclovir and maribavir. Rev Med Virol 2008;18:233-246.

13 Scott GM, Weinberg A, et al: Multidrug resistance conferred by novel DNA polymerase mutations in human cytomegalovirus isolates. Antimicrob Agents Chemother 2007;51:89-94.

14 Sheth A, Boktor M, Diamond K, Lavu K, Sangster G: Complete esophageal obliteration secondary to cytomegalovirus in AIDS patient. Dis Esophagus 2010;23:E32-E34.

15 Dieterich DT, Poles MA, et al: Foscarnet treatment of cytomegalovirus gastrointestinal infections in acquired immunodeficiency syndrome patients who have failed ganciclovir induction. Am J Gastroenterol 1993;88:542-548. 